\title{
TRANSVERSE BEHAVIOUR OF THE LHC PROTON BEAM IN THE SPS: AN UPDATE
}

\author{
G. Arduini, K. Cornelis, W. Höfle, G. Rumolo, F. Zimmermann \\ SL Division, CERN, Geneva, Switzerland
}

\begin{abstract}
During the 1999 SPS run, strong transverse instabilities were observed with the LHC beam [1]. Both the instability characteristics and the identical threshold current as for beam-induced electron multipacting led to consider the interaction of the beam with the electron cloud as a likely source. In 2000, we have measured the dependence of beam motion, beam loss, and emittance growth on bunch intensity, number of bunches, octupole strength, chromaticity, and gaps in the bunch train. We report on these recent studies and compare the beam observations with simulations of electron cloud build up and electron-induced single-bunch instabilities.
\end{abstract}

\section{INTRODUCTION}

The measurements performed in 1999 with the LHC beam [2] evidenced an important blow-up of the tail of the batch in both planes accompanied by a strong horizontal transverse instability developing just after injection. The arguments in favour of an electron cloud instability were:

- similar threshold for the onset of the instability and for the electron cloud, as evidenced by vacuum pressure and pick ups $\left(\mathrm{I}_{\text {bunch }}=4-5 \times 10^{10} \mathrm{p}\right)$.

- increase of the growth rate of the instability along the batch (from the head to the tail) and saturation in the second half of it.

\section{OBSERVATIONS WITH LHC BEAM}

During the year 2000 the electron cloud instability has been studied [3][4][5] under the following conditions:

- $\xi=(\Delta \mathrm{Q} / \mathrm{Q}) /(\Delta \mathrm{p} / \mathrm{p})$ low and positive $(+0.02-+0.05)$.

- Detuning with amplitude minimised by means of machine octupoles.

- Residual coupling: closest-tune approach $\sim 0.001$.

- Transverse feedback active in both planes.

Most the observations discussed in this report were performed at injection in the SPS $(26 \mathrm{GeV} / \mathrm{c})$ and with $\mathrm{I}_{\text {bunch }} \sim 8 \times 10^{10} \mathrm{p}$ (i.e. about $75 \%$ of the nominal intensity and above the threshold for the onset of the beam induced electron multipacting). In such conditions losses occur approximately $4 \mathrm{~ms}$ after injection affecting only the tail of the batch.

\subsection{Transverse motion}

Measurements of the position of the centroid of each bunch of the LHC batch have been performed. The trailing bunches of the batch start to oscillate vertically
30-40 turns after injection and horizontally about 80 turns after injection. The amplitude of the oscillations of the tail of the batch reaches its maximum approximately 150 turns (i.e. $3.5 \mathrm{~ms}$ ) after injection, when the losses occur. The oscillation develops later and later going from the tail to the head of the batch and no significant oscillation is visible for the first 15 bunches.

In the horizontal plane mainly coupled-bunch modes up to about $4 \mathrm{MHz}$ are excited, in the vertical plane higher order modes are also visible. The spectrum observed in the vertical plane is compatible with uncorrelated single bunch dipolar oscillations at a frequency $\left(n+q_{v}\right) f_{r e v}$.

In order to better characterize the vertical instability measurements have been performed with a wide-band transverse pick-up. Figure 1 shows the details of the sum and delta signals for the vertical plane. The signal and the reflection cannot be fully resolved because of the limited length of the strip-line coupler (about $37 \mathrm{~cm}$ ). More intense bunches are more unstable, furthermore the motion of the individual bunches seems to be uncorrelated. The comparison of the spectra of the delta and sum signals also seems to indicate the presence of transverse motion inside the bunch at frequencies of a few hundred MHz. The above behaviour is not observed in the horizontal plane.

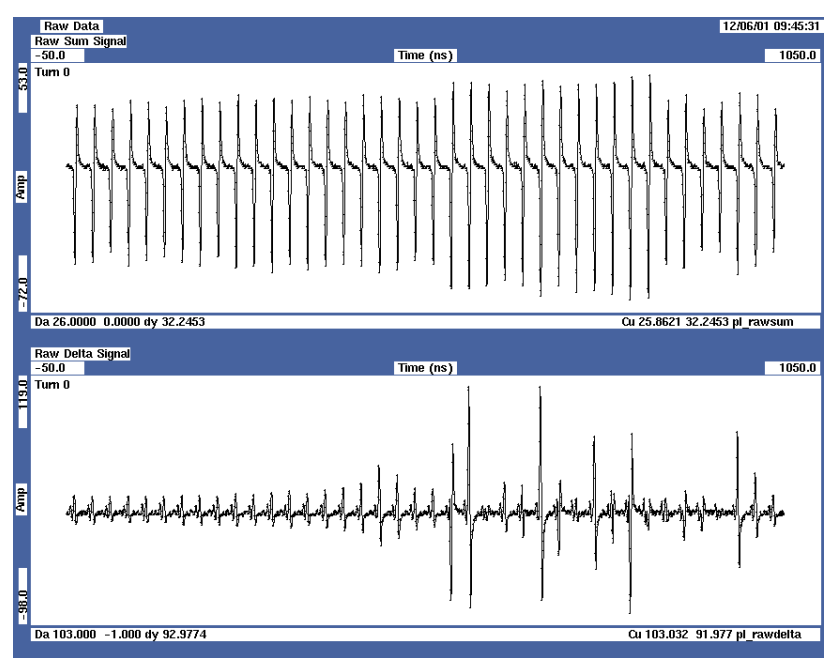

Figure 1: Detail of the sum (top) and delta (bottom) signals provided by the wide-band transverse pick-up (vertical plane). The head of the batch is on the left.

From the data measured with the transverse pick-up the evolution of the phase difference between head and tail of bunches at the beginning and at the end of the LHC batch could be compared. The leading bunches in the batch see the same wake-field as a single bunch, i.e. with an 
interaction length equal or larger than the bunch length. The trailing bunches see a wake-field with an interaction length 0.3 to 0.5 times the bunch length.

The vertical tune increases by more than 0.01 between the $25^{\text {th }}$ and the $30^{\text {th }}$ bunch (see fig. 2). No appreciable shift is observed in the horizontal plane.

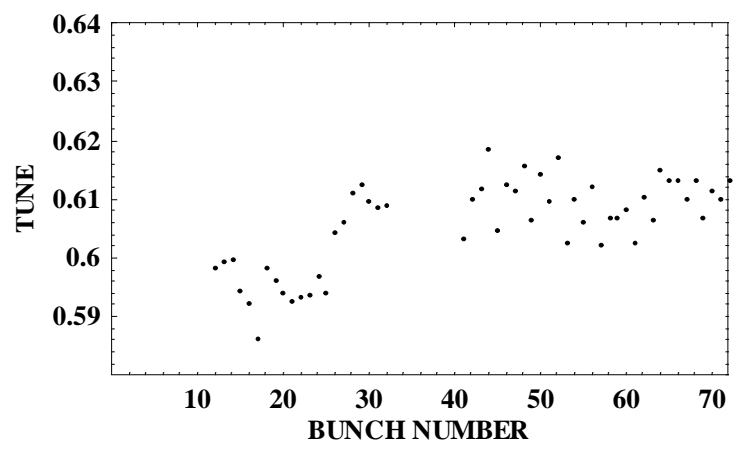

Figure 2: Evolution of the vertical tune along the batch.

Simulations of electron-cloud build up were performed following the recipe described in [6]. Good agreement with observations is obtained if a substantial component of elastically scattered low-energy electrons is included. The measured vertical tune shift of 0.01 is consistent with the simulated electron density of $10^{12} \mathrm{~m}^{-3}$. This tune-shift appears to be 'incoherent' in origin (i.e., resembling an additional quadrupole) as it has opposite sign compared with the tune-shift expected for the electron-cloud short range wake-field measured in the vertical plane.

For a uniform electron cloud in a flat chamber the horizontal tune shift should indeed be much smaller than the vertical, $\Delta \mathrm{Q}_{\mathrm{H}} \sim\left(\mathrm{h}_{\mathrm{V}} / \mathrm{h}_{\mathrm{H}}\right) \Delta \mathrm{Q}_{\mathrm{V}} \sim 0.3 \Delta \mathrm{Q}_{\mathrm{V}}$, where $\mathrm{h}_{\mathrm{H}}$ and $\mathrm{h}_{\mathrm{v}}$ denote the chamber horizontal and vertical dimensions, respectively.

\subsection{Emittance blow-up}

The single-bunch instability driven by the electroncloud wake-field is expected to produce emittance growth in both planes [7][8]. Profile measurements performed at injection (integrated over a few $\mathrm{ms}$ ) indicate a significant blow-up (by more than a factor 2.5 in the horizontal plane and by a factor 4 in the vertical plane) of the tail of the batch. The blow-up is visible after about 15 bunches. In the vertical plane the tail fills-up the physical aperture of the machine producing beam losses. The results of simulations [8] of the growth rate of the single-bunch instability and of the induced emittance blow-up (Fig. 3) are consistent with the observations.

\section{DEPENDENCE ON BUNCH LENGTH}

A qualitative dependence of the electron cloud density on bunch length has been established by observing the baseline drift induced by the electron cloud on the signal provided by electrostatic pick-ups using high impedance electronics for read-out [5]. The measurements were done during the first few ms after injection with a mismatched $\mathrm{RF}$ voltage when significant bunch length oscillations at twice the synchrotron frequency are observed. A significant distortion of the baseline signal is visible when the bunch length is minimum and it disappears after a quarter of a synchrotron period when the bunch length is maximum.
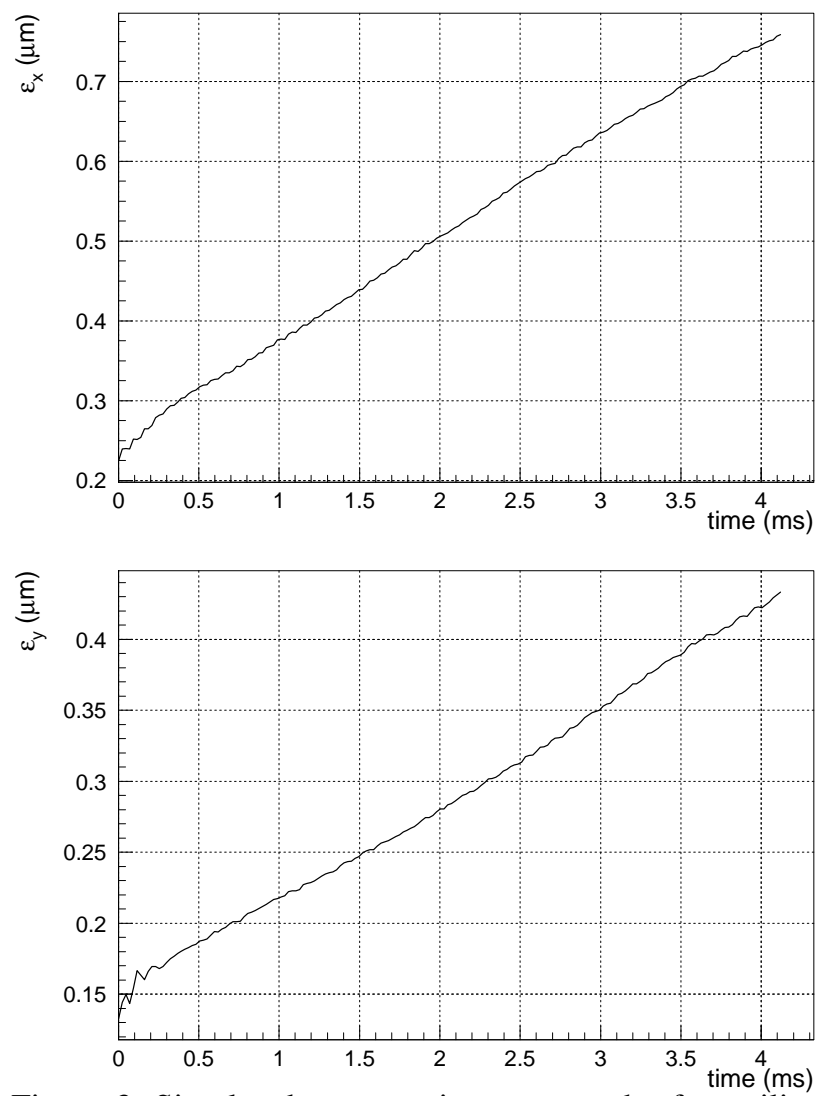

Figure 3: Simulated r.m.s. emittance growth of a trailing bunch due to electron cloud over the first $4 \mathrm{~ms}$ [8].

\section{DEPENDENCE ON THE FILLING PATTERN}

The nominal LHC filling scheme foresees the injection in the SPS of 3 or 4 LHC batches spaced by 225 ns (i.e. a gap of 8 bunches). It is therefore interesting to verify if the above spacing is sufficient to completely uncouple the batches with respect to the beam induced electron cloud. A gap of 12 bunches after 36 bunches was created in the batch. No relevant losses are detectable in the tail of the batch. The bunch-to-bunch centroid motion has been measured. The behaviour of the head of the batch is similar to that observed for the LHC batch without gap. The part of the batch following the gap behaves like the head of the batch but the oscillations start after 10 bunches while in the head of the batch they are visible only after 15 bunches. A gap of 12 bunches after 36 bunches is not sufficient to completely clear off the electron cloud generated by the head of the batch. Similar conclusions can be drawn from the evolution of the vertical tune along the LHC batch. The above behaviour agrees with the electron signal measured by an electron collector installed in a section of the SPS vacuum chamber [9] and also with simulations (Fig.4). A complete reset of the electron-cloud generated by the tail 
of the batch occurs only when a gap of 24 bunches is created after 36 bunches.

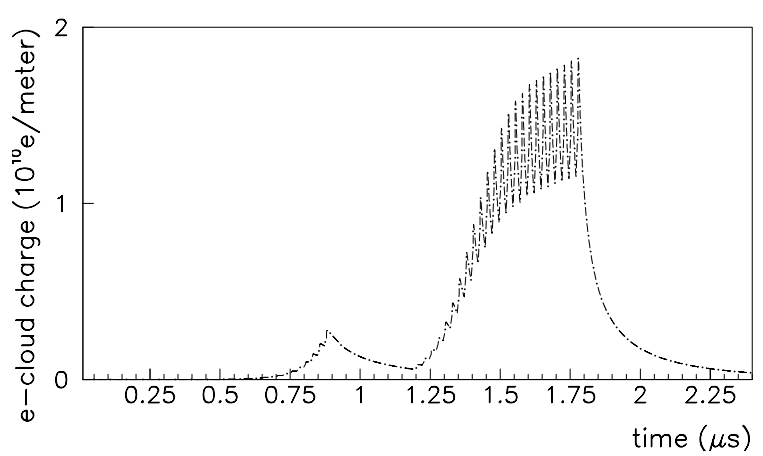

Figure 4: Simulated evolution of the electron line density in units of $10^{10} \mathrm{~m}^{-1}$ as a function of time in $\mu$ s, during the passage of an LHC batch through an SPS dipole chamber with a 12-bunch gap after 36 bunches.

\section{EFFECT OF OCTUPOLES AND CHROMATICITY}

The effect of octupoles and chromaticity as means to fight the electron cloud instability has been investigated.

\subsection{Octupoles}

The dependence of the horizontal and vertical beam size along the batch at injection on different octupole settings has been measured for the working point $\mathrm{q}_{\mathrm{H}}=0.635, \mathrm{q}_{\mathrm{v}}=0.567$ with no transverse feedback. The tail of the batch blows up significantly in the horizontal plane for high radial strengths with the result of stabilising the beam and of eliminating the vertical blow-up. Nevertheless the tail is filling all the available horizontal aperture of the machine and the beam suffers slow losses all through the injection plateau.

\subsection{Chromaticity}

Chromaticity has proven to be the only tool allowing to control the blow-up of the size of the tail of the batch and to obtain a decent lifetime in the injection plateau. The chromaticity values required to stabilise the beam increase with the number of bunches for a given bunch intensity. For the nominal LHC batch (72 bunches) $\xi_{\mathrm{H}}$ up to +0.9 and $\xi_{\mathrm{v}}$ up to +0.5 must be applied. The resulting tune spread prevents a stable operation at the standard working point $\left(\mathrm{q}_{\mathrm{H}}=0.62, \mathrm{q}_{\mathrm{v}}=0.58\right)$ and higher tunes must be chosen $\left(\mathrm{q}_{\mathrm{H}}=0.78, \mathrm{q}_{\mathrm{v}}=0.60\right)$ in order to avoid crossing resonances with part of the beam. Under these conditions no loss is detectable.

The elimination of the blow-up and of the losses at high chromaticities corresponds to the suppression of the described transverse motion.

\section{CONCLUSIONS}

Fast transverse instabilities affect the trailing bunches of the LHC beam in the horizontal and vertical plane. Several arguments seem to indicate the beam induced electron cloud as a source of the observed transverse instabilities:

- Similar threshold for the onset of instability and electron cloud (both in terms of bunch intensity and number of bunches).

- Compatibility of the observations with the measurements and simulations of the electron-cloud build-up and of the induced 'incoherent' tune shift. This is particularly true if a substantial component of elastically scattered low-energy electrons is included.

- Observation of high frequency (single bunch) activity. The unstable frequencies are comparable with the estimated electron oscillation frequency of 200-400 MHz.

The electron cloud acts as a short-range $(0.3-0.5$ times the bunch length) wake-field in the vertical plane and as a longer range (several bunch spacings) wake-field in the horizontal plane. As a result of that the tail of the batch blows up until the vertical physical aperture of the machine is reached and beam losses occur. Neither oscillation nor blow-up is detectable for the first 15 bunches. This excludes resistive wall as a possible source of the observed behaviour.

An enhancement of the electron cloud is expected for subsequent LHC batches in the SPS if the present filling scheme is maintained.

Chromaticity seems for the moment the only tool available to fight the instabilities and to avoid blow-up and losses. It is not yet clear if the above scheme will be viable for the nominal bunch intensities, for more batches and for the acceleration.

\section{REFERENCES}

[1] A General Parameter List of the LHC proton beam in the SPS can be found at the address: http://sl.web.cern.ch/SL/sli/Parameters/main.htm.

[2] G. Arduini, K. Cornelis, G. Ferioli, L. Jensen, F. Zimmermann, "Transverse Instabilities of the LHC proton beam in the SPS". Proceedings of EPAC2000, p. 341-343.

[3] G. Arduini, "Observations on Transverse Instabilities”, CERN SL/2001-003(DI), pp. 125-134.

[4] K. Cornelis, "Measurement of the Electron Cloud Impedance", CERN SL/2001-003(DI), pp. 163-165.

[5] W. Höfle, "Progress with the SPS damper", CERN SL/2001-003(DI), pp. 117-124.

[6] G. Rumolo et al., "Simulation of the Electron-Cloud Build Up and its Consequences on Heat Load, Beam Stability, and Diagnostics", Phys. Rev. ST Accel. Beams 4 (2001) 012801.

[7] K. Ohmi, F. Zimmermann, "Head-Tail Instability caused by electron Cloud in Positron Storage Rings," Phys. Rev. Lett. 85, p. 3821 (2000).

[8] G. Rumolo, F. Zimmermann, "Simulation of Single Bunch Instabilities Driven by Electron Cloud in the SPS", this conference.

[9] J.M. Jimenez, "SPS vacuum observations and electron scrubbing with LHC beams", CERN SL/2001-003(DI), pp. 160-162. 